\title{
Women Exposed to the Scents of Fertile-Phase and Luteal-Phase Women: Evaluative, Competitive, and Endocrine Responses
}

\author{
Savannah L. Woodward • Melissa Emery Thompson • \\ Steven W. Gangestad
}

Received: 11 July 2014 /Revised: 30 November 2014 / Accepted: 4 December 2014 / Published online: 7 January 2015

C) Springer International Publishing 2015

\begin{abstract}
Men prefer the scents of fertile-phase women to the scents of luteal-phase women. Very little research, however, has examined women's responses to other women's scents. The current research did so by asking female participants to smell one of two sets of t-shirts: One set that was worn by women during the fertile phase of their cycle, and one set that was worn by the same group of women during their luteal phase, and then rate the "pleasantness" of the scent of each shirt. Women preferred the scents of shirts worn during the fertile phase. Evidence suggested that exposure to luteal phase shirts may prime more assertive responses to other young women. In women with high estradiol and low progesterone during the session, testosterone became elevated. A number of possible explanations may account for these effects. Additional research is needed to assess them and establish the robustness of these effects.
\end{abstract}

Keywords Scent $\cdot$ Menstrual cycle $\cdot$ Fertile phase $\cdot$ Luteal phase $\cdot$ Testosterone $\cdot$ Estradiol . Progesterone

\section{Introduction}

In a seminal study, Poran (1994) charted men's attraction to their female partner's scent throughout the course of their menstrual cycle. Men consistently rated their partners' scents to be most attractive in the days immediately prior to ovulation. More recent

\footnotetext{
S. L. Woodward

F. Edward Hérbert School of Medicine, Uniformed Services University of the Health Sciences, Bethesda, MD, USA

M. E. Thompson

Department of Anthropology, University of New Mexico, Albuquerque, NM, USA

S. W. Gangestad $(\bowtie)$

Department of Psychology, MSC03 2220, University of New Mexico, Albuquerque, NM 87131, USA

e-mail: sgangest@unm.edu
} 
research has found, repeatedly, that men not pair-bonded to target females demonstrate the same scent preferences: They prefer the scent of fertile-phase women (Singh and Bronstad 2001; Thornhill et al. 2003; Havlíček et al. 2006; Kuukasjärvi et al. 2004; Miller and Maner 2010; Gildersleeve et al. 2012; for one null result, see Thornhill and Gangestad 1999). These findings need not imply that women advertise their fertility status with scent. Indeed, there exists no evidence that women possess specialized mechanisms for production and dissemination or specialized scents at mid-cycle, as some other animal species do. A more likely explanation is that men detect subtle changes in women's scents that are a by-product of other changes linked with fertility status (e.g., associated with hormones or their physiological effects), and that males have been selected to prefer cues of a fertile status (e.g., Thornhill and Gangestad 2008). This preference is probably not a trait strictly derived in humans per se, as males in some primate species exhibit preferences for the scent of fertile-phase females as well (e.g., Cerda-Molina et al. 2006b; Matsumoto-Oda et al. 2002; Ziegler et al. 1993).

Interestingly, men not only prefer, but produce a physiological response to, the scent of fertile-phase women. Miller and Maner (2010) found that men's testosterone levels rose more following exposure to fertile-phase women's scents compared to exposure to luteal-phase women's scents. (For a failure to replicate, see Roney and Simmons 2012. On similar effects in non-human primates, see Cerda-Molina et al. 2006a).

Two published studies to date has asked whether women differentially evaluate the scents of other women, as they vary as a function of fertility status. Maner and McNulty (2013) found no effect of cycle phase of a shirt donor on women's evaluation of the scent of the shirt, while Kuukasjärvi et al. (2004) found a marginally significant effect such that fertile-phase scents were preferred. Power to detect any effect, however, was very low in both studies, as each of 25 women smelled just one shirt (either one donated by a peri-ovulatory woman or one donated by a woman during the luteal phase) in Maner \& McNulty's study, while just 12 women rated shirts in Kuukasjärvi et al.'s study. Maner and McNulty (2013) nonetheless found that women's testosterone changed as a function of being exposed to the scent of a woman during the fertile or luteal phase; the testosterone of women exposed to the scent of a peri-ovulatory woman remained steady or increased over time, whereas the testosterone of women exposed to the scent of a woman during the luteal phase tended to decrease. Maner and McNulty (2013) speculated that the function of this alteration in testosterone level is to facilitate competition with a reproductively capable female, as testosterone appears to function in some other species (e.g., Beehner et al. 2005), but they did not assess female intrasexual competitiveness in their study. The current study sought to examine, using a more powerful design, women's evaluation of the scents of women associated with the fertile and luteal phases. It also sought to address additional questions pertaining to testosterone changes and their possible function. In particular, we asked the following questions:

First, do women prefer the scents of either fertile-phase or luteal-phase women over the other?

Second, how does exposure to the scents of fertile-phase vs. luteal-phase women affect women's intrasexual competitiveness with other women? As argued by Maner and McNulty (2013), fertile-phase women might be viewed as competitors, and hence their scents might evoke competitive behaviors. Alternatively, scents of 
fertile-phase women might have the opposite effect on competitiveness (e.g., because fertile-phase scents suggest to females that it is a propitious time to reproduce).

Third, do women's testosterone levels change differentially as a function of exposure to fertile-phase and luteal-phase scents? Our sample size of both female scent donors (15 vs. 4) and smellers (58 vs. 25) substantially exceeded sample sizes in Maner and McNulty (2013) study, meaning this study should have possessed greater statistical power to detect meaningful changes in women's responses to female scents.

Fourth, we performed exploratory analyses examining the main effects and moderating effects of shirt-smellers' own hormone levels, with specific emphasis on reproductive hormones (estradiol, progesterone, testosterone) - though cortisol was included as well. Following Maner and McNulty (2013) hypothesis, we might expect an enhanced competitive response to fertile-phase scents from women who are also in their fertile phase. Steroid hormones are also known to affect olfactory function (Moffitt 2003). We asked whether women's responses to fertile-phase and luteal-phase women's scents varied as a function of these markers of fertility status. In so doing, we examined main effects of these hormones on outcomes (e.g., scent attractiveness, intrasexual competitiveness) as well as interactions between the hormone levels and donor phase (fertile vs. luteal). Such interactions reflect the extent to which differential responses to fertile-phase vs. luteal-phase scents are associated with steroid hormone levels.

\section{Methods}

\section{Participants and Procedures}

Fifteen women ages $18-38$ (median age $=19.4, \mathrm{SD}=3.6$ ) donated the t-shirts used in this study. They were not taking hormonal birth control, were not menopausal, pregnant or breastfeeding, and reported not to experience irregular cycles. Participants' selfreported menstrual cycle data (day of the cycle and typical cycle length) were used to estimate two periods, during each of which women were instructed to wear a plain white t-shirt, freshly washed in no-scent detergent, for two nights. One was targeted to be the late follicular phase, within the 5-day fertile window that occurs prior to the day of ovulation. On average, day of ovulation occurs 13-14 days prior to the beginning of the following cycle, with $80 \%$ of luteal phase lengths occurring within a 5-day window (e.g., Baird et al. 1995). Hence, women were instructed to wear shirts donated to the fertile phase condition 15-18 nights prior to their next expected menses. The other was targeted to be the mid-luteal phase, which occurs, on average, 1 week after ovulation. Hence, women were instructed to wear these shirts between 7 to 10 nights prior to anticipated end of the cycle. From these 15 women, we obtained 11 shirts worn by women during the late follicular phase, and 13 worn by women during the luteal phase.

We note that, because phase of the cycle of donors was not definitively confirmed (e.g., through testing of a luteinizing hormone, or LH, surge), we cannot be assured that all shirts were worn during targeted phases. And, even in normal young women, 
anovulation occurs in about $8 \%$ of all cycles (Ahrens et al. 2014). However, as each woman rater was exposed to multiple stimuli of the same type, it is almost certainly the case that women assigned to smell shirts worn during the follicular phase were indeed exposed to more shirts worn during that phase, and women assigned to smell shirts during the luteal phase were exposed to more shirts worn during that phase. As a common set of women donated shirts for both phases, features of the scents due to individual identity, and not varying across phase, should have been controlled.

During these two windows, women were asked to shower with unscented soap provided to them, and to avoid scented deodorant, soaps, and perfumes; all spicy and pungent foods (e.g. jalapeños, coffee, garlic, onions, etc.); sleeping with another person; and having sex with another person (for similar procedures, see Kuukasjärvi et al. 2004; Singh and Bronstad 2001; Thornhill and Gangestad 1999).

After wearing a shirt, participants placed it in an unscented plastic bag, and returned it the morning immediately following to the University of New Mexico's Department of Biology. Shirts were kept frozen at $-20{ }^{\circ} \mathrm{C}$ (e.g., Singh and Bronstad 2001) for up to 2 months prior to use as stimuli. Freezing within this timeframe has not been found to affect features (pleasantness, intensity) of odors (Lenochova et al. 2009).

Sixty-seven women ages $18-55$ (median age $=21.25, \mathrm{SD}=6.5$ ) were recruited to smell t-shirts. Participants were college women recruited from a psychology participant pool, and we explicitly targeted women who were normally ovulating. In return for participation, they were given credit toward meeting a research requirement for a psychology class. Participants were randomly assigned to one of two conditions: Exposure to fertile-phase shirts, or exposure to luteal-phase shirts. Though multiple women were sometimes run during a session, each woman was in her own private room during her smelling and rating of shirts. All sessions were run within a consecutive 3-day period, and shirts were never refrozen after unfreezing.

Participants first completed a questionnaire concerning demographics, past and current hormonal birth control use, pregnancy/breastfeeding status, and menstrual cycle. As steroid hormones affect olfactory processing (Moffitt 2003) and we specifically targeted for recruitment normally ovulating women, women who were currently taking contraceptive hormones (5), had taken them within the past 3 months (1), were pregnant (1) or post-menopausal (1, 56 years old), and were taking other hormonal medications (1) were excluded from primary analyses. Additionally, women who reported inconsistent menstrual cycles (indicative of irregular ovulation), including pre-menopausal and menopausal women, were not included. A sample of 58 women, then, met our criteria for inclusion (mean age $=21.14, \mathrm{SD}=5.98$; range $=18-47$; ages for fertile phase and luteal phase shirt-smellers $=21.33$ [SD=6.1], 20.97 [SD=6.0], $t_{56}=0.23, p=0.82$ ).

Participants were then asked to smell and rate each shirt on a scale from one to ten on "pleasantness" of the odor, where one was "Not at all Pleasant," and ten was "Very Pleasant." Participants were then given a series of questionnaires and personality measures.

Two specific questionnaire measures were of interest in this study.

Intrasexual Competitiveness Women were asked a series of 4 paired questions concerning their responses to another woman following an incident that could potentially provoke an aggressive response. Scenarios were based on similar ones used by 
Griskevicius et al. (2009). One question of the pair concerned the participant's likely response to a "young woman"; the other concerned her response to an "elderly woman". For instance, one question was the following: "You see a young woman [an elderly woman] talking to someone you are romantically involved with. Would you: (a) Glare at her, (b) Enter an argument with her, (c) Do nothing, (d) Other Other scenarios were "A woman accidentally spills a drink on you at a party while you are talking to someone you are attracted to"; "A woman swerves in front of you on the freeway"; "A _ _ woman lets her friend cut in line at the grocery store." Responses were graded from least aggressive/competitive (e.g., in the instance above, "Do nothing") to the most (e.g., "Enter an argument with her"). Open-ended responses ( $22 \%$ of all responses) were coded by an individual blind to the participant's condition (mapped onto the range of scores of explicit scores) and totaled to calculate the participants' overall aggressiveness/competitiveness score, as well as an aggressiveness/competitiveness score for both targets (young vs. elderly woman). To obtain a measure of intrasexual competitiveness with a potential sexual rival, we summed the competitiveness of responses to a young woman and subtracted responses to an elderly woman. In this way, we attempted to control for general aggressivity in response to provocations, and target ones specific to responding to a potential sexual rival. Differences (response to young - response to elderly woman) did not highly covary across items; $\alpha=0.25$.

A number of personality questionnaires were not pertinent to the present research.

\section{Salivary Hormone Assays}

Participants were asked to provide two saliva samples via passive drool, following Salimetrics guidelines (www.salimetrics.com). One sample was provided immediately prior to smelling the t-shirts, and one following completion of all measures (approximately $20 \mathrm{~min}$ following exposure to the scents). Samples were frozen at $20{ }^{\circ} \mathrm{C}$. Assays for testosterone, estradiol, progesterone, and cortisol were conducted in the Hominoid Reproductive Ecology Laboratory, University of New Mexico.

Testosterone, estradiol, and progesterone were analyzed with ELISA kits designed for saliva by Salimetrics (Carlsbad, CA). Cortisol was analyzed using a comparable procedure with reagents and protocols provided by the Clinical Endocrinology Laboratory at the University of California at Davis (Antibody R4866, sensitivity $\sim 16 \mathrm{pg} / \mathrm{ml}$ ). Serial dilutions of a saliva sample demonstrated parallelism with cortisol standard curve $(t=-0.39, p=0.71)$. The interassay CVs for low and high samples, respectively, were: 6.3 and $5.0 \%$ (cortisol), 6.4 and $5.1 \%$ (estradiol), 11.7 and $6.4 \%$ (progesterone), and 15.2 and $6.9 \%$ (testosterone). Intraassay CVs, calculated as the mean CV of duplicate sample determinations, were: $8.4 \%$ (cortisol), $6.3 \%$ (estradiol), $9.4 \%$ (progesterone), and $4.9 \%$ (testosterone).

Across groups exposed to fertile-phase and luteal-phase shirt scents, women's preexposure mean levels of testosterone $\left(52.6[\mathrm{SD}=25.6], 55.8\right.$ [34.8], respectively; $t_{53}=$ $0.38, p=0.71$ ), estradiol (3.14 [1.18], 3.17 [1.41]; $\left.t_{53}=-0.07, p=0.94\right)$, and progesterone (42.7 [41.9], 60.8 [49.0]; $\left.t_{53}=1.46, p=0.15\right)$ did not significantly differ. At a marginal level of significance, the pre-exposure cortisol levels of women who smelled fertile-phase shirts $($ mean $=2620, \mathrm{SD}=1284)$ were less than the pre-exposure levels of 
women who smelled luteal-phase shirts $($ mean $=3511, \mathrm{SD}=2220), t_{45.9}=1.83, p=0.073$ (test using separate variance estimates used, as variances significantly differed: Levene's test: $\mathrm{F}_{1.52}=4.31, p=0.043$ ).

\section{Analysis Strategy}

We analyzed data in two steps.

First, we focused on whether exposure to fertile-phase or luteal-phase scents affected women's responses. In the cases of scent attractiveness and intrasexual competitiveness, we performed simple t-tests. In the cases of testosterone, we ran an ANCOVA (under SPSS 22.0, GLM univariate) on post-exposure levels as a function of donor phase, with pre-exposure level entered as a covariate.

Second, we explored hormonal main effects and interactions with donor phase. We performed ANCOVAs (under SPSS 22.0, GLM univariate) with (a) donor phase entered as a factor, (b) all pre-exposure hormone levels entered as covariates, and (c) interactions between pre-hormone levels and donor phase entered as well. All covariates were zero-centered through $z$-scoring for these analyses, so that main effects would be interpretable as overall main effects. We followed up significant interactions by examining the effect of donor phase at $1 \mathrm{SD}$ above the mean on pre-exposure hormone level, and 1 SD below the mean on pre-exposure hormone level, as recommended by Cohen et al. (2003).

Estradiol and progesterone levels should not be strongly affected in the short term, as levels should largely be produced in the granulosa cells in the ovarian follicles or the corpus luteum, not strongly under immediate neural control. To check this assumption, we ran repeated measures ANOVAs on pre- and post-exposure levels of these hormones, with donor phase entered as a between-subject factor. For estradiol, pre- and post- levels did not differ, $F_{1,51}=0.00, p=0.95$, eta ${ }^{2}=0.000$, and change was not significantly moderated by donor phase, $F_{1,51}=2.05, p=0.158$, eta ${ }^{2}=0.039$. Progesterone levels were higher post-exposure than pre-exposure, $F_{1,51}=4.39$, $p=0.041$, eta ${ }^{2}=0.079$, though change was not moderated by donor phase, $F_{1,51}=$ $0.45, p=0.51$, $\mathrm{eta}^{2}=0.009$. An examination of change scores revealed, however, that the effect was driven by three outlying cases, all $>7 \mathrm{SD}$ from the mean when they were excluded, > 3.5 SD from the next highest value. For inexplicable reasons, we had measured very low initial levels of progesterone in these women, but much higher levels later in the session. With these outliers eliminated, pre- and post-exposure levels did not differ, $F_{1,51}=1.07, p=0.31$, eta ${ }^{2}=0.022$, and the correlation between them was very high, $r=0.85, p<0.000001$. (With outliers included, the correlation was 0.51 .) To obtain more reliable measures of estradiol and progesterone, under the assumption that they were not meaningfully affected by the manipulation, we averaged pre- and postlevels, with mean substitution of progesterone values for the three women with outlying change scores. Results were very similar when we excluded these three cases or retained their mean scores.

In contrast to estradiol and progesterone, we used the pre-exposure values for cortisol and testosterone in all analyses, as those hormones might be systematically affected by exposure to scents.

Correlations between before- and after-exposure levels of testosterone, estradiol, and cortisol were $0.58(p<0.001), 0.28(p<0.05)$, and $0.60(p<0.001)$, respectively. The 
correlation for estradiol was suppressed by one apparent bivariate outlier; with that woman removed, the correlation was $0.41, p=0.003$. Her mean value, used in the analysis, however, was very close to the mean. We retained that value in all analyses.

\section{Results}

\section{Women's Scent Preferences}

Fertile-Phase vs. Luteal Phase Scents With a simple $t$-test, we assessed whether women's mean scent attractiveness ratings differed as a function of donor phase (fertile vs. luteal). In fact, women's mean ratings of fertile-phase shirts significantly exceeded their mean ratings of luteal phase shirts, means $=4.97(\mathrm{SD}=1.41)$ vs. $4.24(\mathrm{SD}=1.27)$, $t_{56}=2.07, p=0.043$.

The Effects of Rater Hormone Levels In follow-up analyses adding rater hormone levels as predictors, donor phase significantly affected scent attractiveness, $F_{1,44}=$ 7.31, $p=0.010$, partial eta ${ }^{2}=0.142$. In addition, rater's cortisol had a significant mean positive effect on ratings across conditions, $F_{1,44}=4.09, p=0.049$ partial eta ${ }^{2}=0.085$; testosterone had a marginally significant positive effect, $F_{1,44}=4.02, p=0.051$, partial eta $^{2}=0.082$.

The main effect of cortisol, however, was qualified by a significant interaction with donor phase, $F_{1,44}=4.77, p=0.034$, partial eta ${ }^{2}=0.098$. To interpret this interaction, we conducted analyses assessing simple main effects of donor phase at one standard deviation above and one standard deviation below the mean on cortisol (as recommended by Cohen et al. 2003). At one standard deviation above the mean on cortisol, donor phase had a significant effect on scent attractiveness, $F_{1,44}=9.82, p=$ 0.003 , partial eta ${ }^{2}=0.182$, with fertile-phase shirts being preferred. No effect of phase emerged at one standard deviation below the mean on cortisol, $F_{1,44}=0.00, p=0.95$, partial eta ${ }^{2}=0.000$.

With all non-significant terms removed, the cortisol $\mathrm{x}$ donor phase interaction remained significant, $F_{1,49}=4.29, p=0.044$, partial eta ${ }^{2}=0.080$.

\section{Women's Intrasexual Aggressiveness}

Fertile-Phase vs. Luteal-Phase Scents We performed a similar analyses on women's aggressiveness toward a young (vs. older) woman. A $t$-test yielded a significant effect of donor phase, $t_{56}=2.10, p=0.041$. On average, women exposed to luteal-phase shirts expressed greater levels of assertiveness in the test scenario involving a hypothetical young woman (relative to an elderly woman), compared to women exposed to fertilephase shirts, means $=0.38(\mathrm{SD}=0.33)$ and $0.21(\mathrm{SD}=0.26)$, respectively. Our measure of intrasexual competitiveness may be interpreted as an ordinal rather than intervallevel scale (given the ordinal nature of increases in competitiveness of possible responses). In an ordinal logistic regression treating intrasexual competitiveness as reflecting ordered responses, the effect of donor phase was marginally 
significant, Wald $=3.41, p=0.065$. A Mann-Whitney $\mathrm{U}$ non-parametric test yielded exactly the same $p: 0.065$.

The Effects of Rater Hormone Levels In a follow-up ANCOVA with hormone levels added, effect of donor phase fell short of significance, $F_{1,44}=3.86, p=0.056$, partial $\left.\mathrm{eta}^{2}=0.081\right)$. No main effects or interactions involving hormone levels were statistically robust, all $p \geq 0.146$.

\section{Post-Scent Exposure Testosterone Levels}

Fertile-Phase vs. Luteal-Phase Scents As expected, pre-exposure testosterone levels, entered as a covariate, strongly predicted post-exposure levels, $F_{1,50}=25.81, p<0.001$, partial eta ${ }^{2}=0.340$. We detected no effect of donor phase, $F_{1,50}=1.42, p=0.239$, partial eta $^{2}=0.028$; marginal means for fertile-phase and luteal-phase conditions $=56.92(\mathrm{SE}=$ 3.66), $50.92(\mathrm{SE}=3.46)$, respectively.

The Effects of Rater Hormone Levels To examine whether rater hormone levels moderated the testosterone response to a donor scent, we did not include preexposure testosterone itself as a moderator but rather focused on moderating influences of the other hormones (but see our follow-up analysis below). The effect of donor phase on testosterone response was marginally significant, $F_{1,43}=3.10, p=0.085$, partial eta ${ }^{2}=$ 0.067 . But, this effect was significantly moderated by both estradiol, $F_{1,43}=6.55, p=$ 0.014 , partial eta ${ }^{2}=0.132$; and progesterone, $F_{1,43}=6.04, p=0.018$, partial eta ${ }^{2}=0.123$.

At one standard deviation above the mean in estradiol levels, donor phase had a large effect, $F_{1,43}=9.19, p=0.004$, partial eta ${ }^{2}=0.176$. Women exposed to fertile-phase shirts had higher testosterone compared to women exposed to luteal-phase shirts. At one standard deviation below the mean in estradiol, no effect of donor phase emerged, $F_{1,43}=0.62, p=0.43$, partial eta ${ }^{2}=0.014$. Figure 1 illustrates this interaction.

At one standard deviation below the mean in pre-scent progesterone levels, donor phase had a robust effect, $F_{1,43}=8.39, p=0.006$, partial eta ${ }^{2}=0.163$. Once again, women exposed to fertile-phase shirts had higher testosterone levels relative to women exposed to luteal-phase shirts. At one standard deviation above the mean in progesterone, no effect of phase was detected, $F_{1,43}=0.55, p=0.51$, partial eta $^{2}=0.010$. See Fig. 2.

With cortisol and its interaction with donor phase eliminated from the model (as neither approached significance), the interaction between donor phase with estradiol remained significant, $F_{1,46}=4.38, p=0.042$, partial eta ${ }^{2}=0.087$, and the interaction between donor phase and cortisol was marginally significant, $F_{1,46}=3.36, p=0.073$, partial eta ${ }^{2}=0.068$.

One can also consider the joint hormonal effects. When women had hormone levels characteristic of the late follicular phase - one standard deviation above the mean on estradiol and one standard deviation below the mean on progesterone - the effect of donor phase was especially pronounced, $F_{1,43}=11.82, p=0.001$, partial eta ${ }^{2}=0.216$. Again, women exposed to fertile-phase shirts had higher testosterone levels relative to women exposed to luteal-phase shirts. This effect remained robust with non-significant cortisol effects removed from the model, $F_{1,46}=8.08, p=0.007$, partial eta ${ }^{2}=0.149$. 


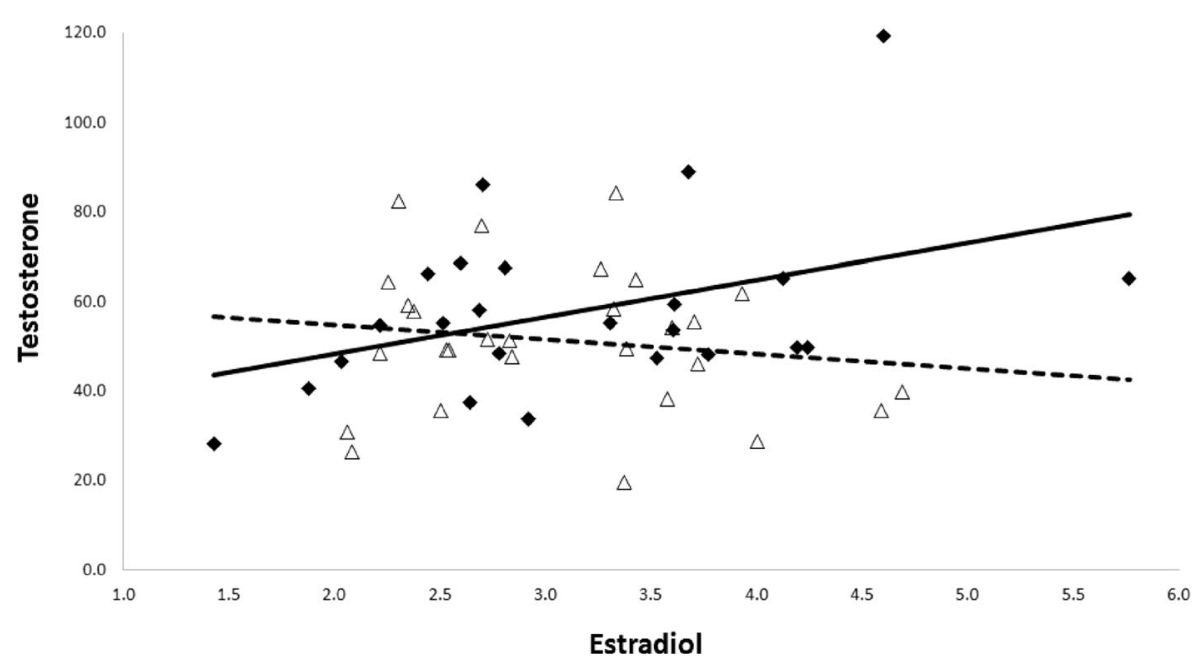

Fig. 1 Association between estradiol (pg/ml) during the session and testosterone $(\mathrm{pg} / \mathrm{ml})$ after exposure to scents as a function of donor phase. Black diamonds and solid regression line: Fertile phase scents. Open triangles and dotted regression line: Luteal phase scents. Values calculated as residuals with all other predictors partialled out, mean centered

When testosterone was also included as a moderator of the effects of donor phase on testosterone itself, its effect fell short of significance, $F_{1,42}=3.76, p=0.059$, partial eta $^{2}=0.082$. The effect of donor phase was not significant, $F_{1,42}=2.64, p=0.111$, partial eta $^{2}=0.059$. The interaction between estradiol and donor phase became marginally significant, $F_{1,42}=3.80, p=0.058$, partial eta ${ }^{2}=0.083$. Nonetheless, the effect of donor phase remained pronounced when women were one standard deviation above the mean on estradiol and one standard deviation below the mean on progesterone, with or

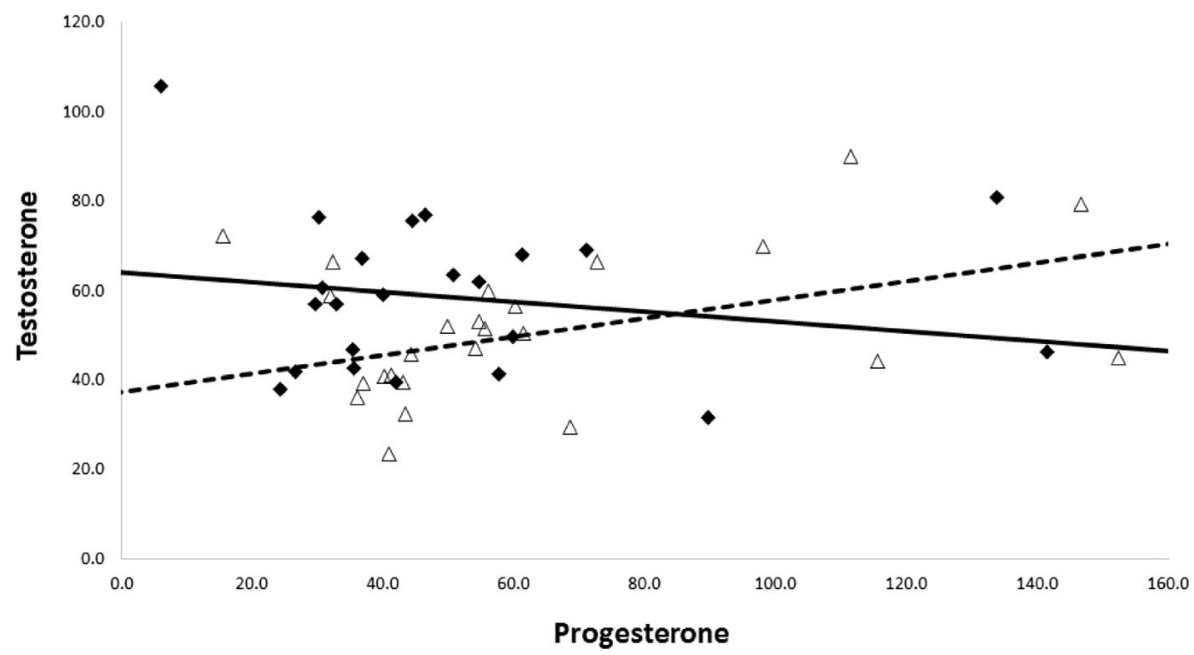

Fig. 2 Association between progesterone (pg/ml) during the session and testosterone (pg/ml) after exposure to scents as a function of donor phase. Black diamonds and solid regression line: Fertile phase scents. Open triangles and dotted regression line: Luteal phase scents. Values calculated as residuals with all other predictors partialled out, mean centered 
without cortisol effects in the model: $F_{1,42}=9.49, p=0.004$, partial eta ${ }^{2}=0.184$, and $F_{1,45}=8.55, p=0.005$, partial eta ${ }^{2}=0.160$, respectively.

\section{Post-Scent Exposure Cortisol Levels}

We performed exploratory analyses examining effects of donor phase and interactions with hormone levels on post-exposure cortisol levels. With pre-exposure cortisol levels controlled, the effect of donor phase was not significant, $F_{1,53}=1.85, p=0.18$, partial $\mathrm{eta}^{2}=0.034$. No interactions between donor phase and hormone levels were detected, whether the interaction with pre-exposure cortisol level was included in the model or not, all $F_{1,41 / 42}=1.14, p>0.29$. One main effect emerged: Estradiol levels positively predicted post-exposure cortisol levels, $F_{1,42}=5.62, p=0.022$, partial eta ${ }^{2}=0.118$. This effect may not be robust, as it was the sole effect, of 7 examined, that emerged.

\section{Discussion}

\section{Summary of Findings}

In this study, we explored answers to a number of questions.

First, do women evaluate the scents of other women differently, depending on the cycle phase of target women? The answer appears to be yes. Just as men do, women in our study found the scents of fertile-phase women to be more attractive than the scents of luteal-phase women. This effect emerged in a simple $t$-test comparing the effects of donor phase on scent attractiveness ratings, and was especially strong after controlling for effects of potential hormonal effects.

Second, does exposure to scents of fertile-phase and luteal-phase women differentially prime intrasexually-competitive motives in women? The answer is a qualified yes. We found that, in response to scenarios involving a young woman (in comparison to an older woman), women in our study responded in more aggressive, competitive ways following exposure to luteal-phase scents, relative to exposure to fertile-phase scents. We do stress, however, that in non-parametric tests applied because our measure of intrasexual competitiveness may offer ordinal but not interval-level data, the effect fell short of significance $(p=0.065)$. Nonetheless, our results may be of interest because we distinctly found no evidence in favor of Maner and McNulty's (2013) proposal, which is that exposure to fertile-phase scents leads women's intrasexual competitiveness to increase; effects in our study ran in the opposite direction. One might be concerned that our measure of intrasexual aggressiveness toward a young potential competitor had low reliability and, potentially validity, despite a very similar measure having been used in previous research (Griskevicius et al. 2009); square root of reliability (estimated $r$ with "true scores") was just 0.50 . Nonetheless, even if low reliability compromised power to detect effects, effects opposite prediction should not be expected.

Third, do women's testosterone levels respond differentially as a function of exposure to other women's scents during the fertile phase as opposed to scents during the luteal phase? Maner and McNulty (2013) found that women's testosterone levels rise in response to fertile-phase scents more so than luteal- 
phase scents. In our analyses, this main effect fell short of significance (though was marginally significant in one analysis). Strikingly, however, we found evidence for interactions between phase of donor scent and reproductive hormone levels. In particular, women's testosterone levels were especially high following exposure to fertile (vs. luteal) phase scents when estradiol levels were high and progesterone low - conditions that, themselves, are characteristic of the fertile phase (e.g., Roney and Simmons 2013). When testosterone itself was added as another moderator, both its moderation effect and that of estradiol became marginally significant. Yet, as both effects were in the same direction and both estradiol and testosterone tend to be elevated in the late follicular phase (Roney and Simmons 2013), the general point holds. Specifically, our findings qualify Maner and McNulty's (2013): Exposure to the scent of fertilephase shirts may prompt a testosterone response in women, but selectively in women whose hormone profile is characteristic of the fertile phase.

In addition, we found a moderating effect of cortisol levels on effects of donor phase on scent attractiveness. Fertile-phase scents were particularly preferred by women with relatively high levels of cortisol.

\section{Robustness of the Interactions}

As noted in the introduction, our examination of the moderating effects of women's own hormone levels on their responses to women's scents was exploratory. Steroid hormones are widely recognized to affect olfactory processing and, hence, such moderating effects are very plausible. At the same time, as it is not clear precisely what chemicals in women's scents women might respond to, we offered no a priori predictions. A number of moderating effects emerged.

One may wonder how robust these effects are. We examined moderating effects of 4 hormone levels on 3 primary outcomes of interest: scent attractiveness, intrasexual competitiveness, and testosterone - for a total of 12 effects. Three, or $25 \%$, emerged as significant at the 0.05 level -5 times the number expected by chance alone. At the same time, none would be significant if a very conservative Bonferroni adjustment for multiple comparisons were made: under that criterion, significant $p$ s would be less than $0.004(0.05 / 12)$, whereas observed $p$-values were $0.034,0.014$, and 0.018 . Simple main effects at levels of the reproductive hormones characteristic more of the fertile than luteal phase (one standard deviation above the mean on estradiol and testosterone, one standard deviation below the mean on progesterone) were more impressive. Two simple effects were significant, with $p$ values being 0.004 and 0.006 . The combination of high estrogen and low progesterone yielded a simple main effect of donor phase on post-exposure testosterone at $p=0.001$.

Naturally, we cannot say with certainty that the effects emerging in the exploratory component of this study are robust and generalizable. We consider them provisional and strongly suggestive - but replication is needed.

\section{Interpretations}

What explains effects of women's fertile-phase and luteal-phase scents on women's responses? At least three scenarios can be envisioned. 
First, women's responses to female scents may themselves reflect adaptation. One must ask, why would it have been adaptive for human females to selectively prefer the scents of fertile-phase females (or, conversely, find the scents of women during luteal phase more aversive)? And why would they respond less aggressively following exposure to these scents (or, conversely, more aggressively following exposure to luteal phase scents)? One possibility is that these responses reflect female interest in other women's fertile status. Some studies have hypothesized that olfactory or pheromonal cues may facilitate reproductive synchrony (Stern and McClintock 1998; Morofushi et al. 2000; Preti et al. 1986). One possible benefit then is that women are signaled by fertile-phase potential competitors that now is the time to reproduce. Alternatively, synchrony may interfere with the ability of dominant males to successfully compete for fertile-phase females, which may be advantaged in a species with male care. Evidence for the presence of this phenomenon in humans, and whether it is mediated by olfaction, however, is highly debated (e.g., Wilson 1992; Weller and Weller 1993; Schank 2006).

Second, women's responses to female scents may be byproducts of adaptations that men possess. Men prefer women's fertile-phase scents. Perhaps women do too, for the same reason that, for example, men have nipples: Though not adaptive traits in a sex themselves, these traits are expressed in the other sex due to lack of fully effective sex limitation.

Third, women's responses to female scents may be byproducts of adaptations that women have with respect to men's scents. During the fertile phase of their cycles, women appear to find most appealing the scent of men who exhibit developmental stability (Gangestad and Thornhill 1998; Thornhill and Gangestad 1999; Thornhill et al. 2003), social dominance (Havlíček et al. 2005), or high levels of testosterone (Thornhill et al., 2013; cf. Rantala et al. 2006). The biochemical correlate(s) of these features that women respond to remains unknown, but candidates include androgens secreted in axillary regions, including androstenol ( $5 \alpha$-androst-16-en-3 $\alpha$-ol), which has a generally pleasant, musky scent (see Gower and Ruperelia 1993, for a review). Androstenone ( $5 \alpha$-androst-16-en-3-one), which typically has a more pungent scent, is produced when androstenol is oxidized (Gower and Ruperelia 1993). Both qualify as 16-androstenes. During the fertile phase, women may be more sensitive to musk-like scents (e.g. Doty 1981; Vierling and Rock 1967; although see Amoore et al. 1977; see also Pause et al. 1996) and find androstenone less aversive (Grammer 1993; Hummel et al. 1991). Perhaps responses to these androgens underlie preference shifts for scents of men with specific phenotypes (though why these scents would be associated with male developmental stability or dominance remains unclear).

Women excrete, on average, only about one-third the levels of androstenol that men do (e.g., Brooksbank 1962). Their concentrations of androstenol in axillary extracts, however, have been reported to change across the ovulatory cycle, peaking during the mid-follicular phase (Preti et al. 1987). In principle, then, concentrations could have been greater in the fertile-phase scents compared to the luteal-phase scents used in this study. Perhaps as byproduct of women's adaptive preference for male androstenol, women also find more appealing the scents of women with greater concentrations of androstenol.

This possibility might also explain why exposure to fertile-phase scents may lead women to react less aggressively to a potential rival in hypothetical scenarios. Benton 
(1982) exposed women to the scent of androstenol across the cycle, and found that androstenol exposure on fertile days led to decreases in aggressive mood. Functionally, this effect has been understood in terms of promoting affiliation in response to the scent of male androstenol (e.g., Benton 1982). Given that there are a large number of highlycorrelated changes in hormone production across the ovulatory cycle, however, there are a large number of possible contributors to variation in scent.

One intriguing set of results in this regard concerns the effect of donor phase on testosterone. Again, though provisional, our findings suggest that women with high levels of estradiol and low levels of progesterone responded to fertile-phase scents with increases in testosterone. Might these changes be byproducts of female responses to male scents as well, with increases facilitating mating efforts and interests specifically when women are fertile (e.g. Bancroft et al. 1991; Bui et al. 2013; cf. Roney and Simmons 2013)? We offer this suggestion cautiously, as moderating effects of hormones require replication.

Naturally, the first two scenarios listed above cannot be ruled out. More data are needed to establish whether hormones are robust moderators of women's ratings of other women's scents, and if so if this is due to a generalized sensory enhancement or to selective preferences for particular scents. Future research might also examine a fuller range of women's psychological responses to female scents (e.g., their sexual interests). Finally, missing links in studies of both male and female responses to fertile-phase women (or, for that matter, developmentally stable or socially dominant men) are the chemicals that mediate these responses. Are they 16-androstenes, or something else? To assess these mediators, future research should measure chemical extracts from shirts or pads worn by targets and evaluated by perceivers (see also Havlíček et al. 2010).

\section{Summary}

This study is one of the first to examine women's affective responses to other women's scents. We find that women, like men, prefer the scent of fertile-phase women to that of luteal-phase women. Evidence suggests that exposure to luteal-phase scents may be associated with greater assertiveness toward other women of reproductive ages. Effects of exposure to these scents on women's testosterone levels may be moderated by women's hormone levels at the time of exposure. Additional research is needed to establish the robustness and appropriate adaptationist interpretation of these phenomena.

\section{References}

Ahrens, K. A., Vladutui, C. J., Mumford, S. L., Schliep, K. C., Perkins, N. J., Wactawski-Wende, J., \& Schisterman, E. F. (2014). The effect of physical activity on reproductive function across the menstrual cycle. Annals of Epidemiology, 24, 127-134.

Amoore, J. E., Pelosi, P., \& Forrester, L. J. (1977). Specific anosmias to 5 alpha-androst-16-en-3-one and alpha-pen- tadecalactone: the urinous and musky primary odours. Chemical Senses and Flavour, 2, 401425 .

Baird, D. D., McConnaughey, R., Weinberg, C. R., Musey, P. I., Collins, D. C., Kesner, J. S., Knect, E. A., \& Wilcox, A. J. (1995). Application of a method for estimating day of ovulation using urinary estrogen and progesterone metabolites. Epidemiology, 6, 547-550. 
Bancroft, J., Sherwin, B. B., Alexander, G. M., Davidson, D. W., \& Walker, A. (1991). Oral contraceptives, androgens, and the sexuality of young-women: II. The role of androgens. Archives of Sexual Behavior, 20, $121-135$.

Beehner, J. C., Philips-Conroy, J. E., \& Whitten, P. L. (2005). Female testosterone, dominance rank and aggression in an Ethiopian population of hybrid baboons. American Journal of Primatology, 67, 101-119.

Benton, D. (1982). Influence of androstenol - a putative human pheromone - on mood throughout the menstrual cycle. Biological Psychology, 15, 249-256.

Brooksbank, B. W. L. (1962). Urinary excretion of androst-16-en-3 alpha-ol in human male axillary sweat. Experientia, 30, 864-865.

Bui, H. N., Sluss, P. M., Blincko, S., Knol, D. L., Blankenstein, M. A., \& Heijboer, A. C. (2013). Dynamics of serum testosterone during the menstrual cycle evaluated by daily measurements with an ID-LC-MS/MS method and a 2nd generation automated immunoassay. Steroids, 78, 96-101.

Cerda-Molina, A. L., Hernandez-Lopez, L., Chavira, R., Cardenas, M., Paez-Ponce, D., Cervantes-de la Luz, H., \& Mondragon-Ceballos, R. (2006a). Endocrine changes in male stumptailed macaques (Macaca arctoides) as a response to odor stimulation with vaginal secretions. Hormones and Behavior, 49, 81-87.

Cerda-Molina, A. L., Hernandez-Lopez, L., Rojas-Maya, S., Murcia-Mejia, C., \& Mondragon-Ceballos, R. (2006b). Male-induced sociosexual behavior by vaginal secretions in Macaca arctoides. International Journal of Primatology, 27, 791-807.

Cohen, J., Cohen, P., Aiken, L. S., \& West, S. H. (2003). Applied regression/correlation analysis for the behavioral sciences. Hillsdale: Lawrence Erlbaum Associates.

Doty, R. L. (1981). Olfactory communication in humans. Chemical Senses, 6, 351-376.

Gangestad, S. W., \& Thornhill, R. (1998). Menstrual cycle variation in women's preference for the scent of symmetrical men. Proceedings of the Royal Society of London B, 265, 927-933.

Gildersleeve, K., Haselton, M. G., Larson, C. M., \& Pillsworth, E. G. (2012). Body odor attractiveness as a cue of impending ovulation in women: evidence from a study using hormone-confirmed ovulation. Hormones and Behavior, 61, 157-161.

Gower, D. B., \& Ruperelia, B. A. (1993). Olfaction in humans with special reference to odorous 16androstenes: their occurrence, perception and possible social, psychological and sexual impact. Journal of Endocrinology, 137, 167-187.

Grammer, K. (1993). 5-“-androst-16-en-3”-on: a male pheromone? A brief report. Ethology and Sociobiology, 14, 201-214.

Griskevicius, V., Tybur, J. M., Gangestad, S. W., Perea, E. F., Shapiro, J. R., \& Kenrick, D. T. (2009). Aggress to impress: hostility as an evolved context-dependent strategy. Journal of Personality and Social Psychology, 96, 980-994.

Havlíček, J., Roberts, S. C., \& Flegr, J. (2005). Women's preference for dominant male odour: effects of menstrual cycle and relationship status. Biology Letters, 1, 256-259.

Havlíček, J., Dvorakova, R., Bartos, L. \& Flegr, J. (2006). Non-advertized does not mean concealed: Body odour change across the human menstrual cycle. Ethology, 112, 81-90.

Havlíček, J., Murray, A. K., Saxton, T. K., \& Roberts, S. C. (2010). Current issues in the study of androstenes in human chemosignaling. Vitamins and Hormones, 83, 47-81.

Hummel, T., Gollisch, R., Wildt, G., \& Kobal, G. (1991). Changes in olfactory perception during the menstrual cycle. Experientia, 47, 712-715.

Kuukasjärvi, S., Eriksson, C. J. P., Koskela, E., Mappes, T., Nissinen, K., \& Rantala, M. J. (2004). Attractiveness of women's body odors over the menstrual cycle: the role of oral contraception and received sex. Behavioral Ecology, 15, 579-584.

Lenochova, P., Roberts, S. C., \& Havlíček, J. (2009). Methods of human body odor sampling: the effect of freezing. Chemical Senses, 34, 127-138.

Maner, J. K., \& McNulty, J. K. (2013). Attunement to the fertility status of same-sex rivals: women's testosterone responses to olfactory ovulation cues. Evolution and Human Behavior, 34, 412-418.

Matsumoto-Oda, A., Oda, R., Hayashi, Y., Murakami, H., Maeda, N., Kumazaki, K., \& Matsuzawa, T. (2002). Vaginal fatty acids produced by chimpanzees during menstrual cycles. Folia Primatologica, 74, 75-79.

Miller, S. L., \& Maner, J. K. (2010). Scent of a woman: men's testosterone responses to olfactory ovulation cues. Psychological Science, 21, 276-283.

Moffitt, C. A. (2003). Steroid hormone modulation of olfactory processing in the context of socio-sexual behaviors in rodents and humans. Brain Research Reviews, 43, 192-206.

Morofushi, M., Shinohara, K., Funabashi, T., \& Kimura, F. (2000). Positive relationship between menstrual synchrony and ability to smell 5alpha-Androst-16-en-3alpha-ol. Chemical Senses, 25, 407-411. doi:10. 1093/chemse/25.4.407. 
Pause, B. M., Sojka, B., Krauel, K., Fehmwolfsdorf, G., \& Ferstl, R. (1996). Olfactory information-processing during the course of the menstrual cycle. Biological Psychology, 44, 31-54.

Poran, N. S. (1994). Cycle attractivity of human female odors. Oxford: Pergamon Press.

Preti, G., Cutler, W. B., Christensen, C. M., Lawley, H., Huggins, G. R., \& Garcia, C. R. (1987). Analysis of compounds from human samples which influence menstrual timing. Journal of Chemical Ecology, 13, 717-731.

Preti, G., Cutler, W. B., Garcia, C. R., Huggins, G. R., \& Lawley, H. J. (1986). Human axillary secretions influence women's menstrual cycles: the role of donor extract of females. Hormones and Behavior, 20, 474 482. doi:10.1016/0018-506X(86)90009-7.

Rantala, M. J., Eriksson, C. J. P., Vainikka, A., \& Kortet, R. (2006). Male steroid hormones and female preference for male body odor. Evolution and Human Behavior, 27, 259-260.

Roney, J. R., \& Simmons, Z. L. (2012). Men smelling women: null effects of exposure to ovulatory sweat on men's testosterone. Evolutionary Psychology, 10, 703-713.

Roney, J. R., \& Simmons, Z. L. (2013). Hormonal predictors of sexual motivation in natural menstrual cycles. Hormones and Behavior, 63, 636-645.

Schank, J. C. (2006). Do human menstrual-cycle pheromones exist? Human Nature, 17, 448-470.

Singh, D., \& Bronstad, P. M. (2001). Female body odor is a potential cue to ovulation. Proceedings of the Royal Society of London B, 268, 797-801.

Stern, K., \& McClintock, M. K. (1998). Regulation of ovulation by human pheromones. Nature, 392, 177179.

Thornhill, R., \& Gangestad, S. W. (1999). The scent of symmetry: a human sex pheromone that signals fitness? Evolution and Human Behavior, 20, 175-201.

Thornhill, R., \& Gangestad, S. W. (2008). The evolutionary biology of human female sexuality. New York: Oxford University Press.

Thornhill, R., Gangestad, S. W., Miller, R., Scheyd, G., Knight, J., \& Franklin, M. (2003). MHC, symmetry, and body scent attractiveness in men and women. Behavioral Ecology, 14, 668-678.

Thornhill, R., Flynn Chapman, J., \& Gangestad, S. W. (2013). Women's preferences for men's scents associated with testosterone and cortisol levels: changes across the ovulatory cycle. Evolution and Human Behavior, 34, 216-231.

Vierling, J. S., \& Rock, J. (1967). Variations in olfactory sensitivity to exaltolide during the menstrual cycle. Journal of Applied Physiology, 22, 311-315.

Weller, L., \& Weller, A. (1993). Human menstrual synchrony: a critical assessment. Neuroscience \& Biobehavioral Reviews, 17, 427-439. doi:10.1016/S0149-7634(05)80118-6.

Wilson, H. (1992). A critical review of menstrual synchrony research. Psychoneuroendocrinology, 17, 565591. doi:10.1016/0306-4530(92)90016-Z.

Ziegler, T. E., Epple, G., Snowdon, C. T., Porter, T. A., Belcher, A. M., \& Küderling, I. (1993). Detection of the chemical signals of ovulation in the cotton-top tamarin, Saguinus oedipus. Animal Behaviour, 45, 313-322. doi:10.1006/anbe.1993.1036. 